COLLOQUIUM MATHEMATICUM

Let us suppose now that II does not hold. Then there exist an $\operatorname{arc} L^{\prime}=(p, q)$ and a sequence of $\operatorname{arcs}\left(u_{i}, v_{i}\right)$ such that $(p, q) \underset{p_{i}}{\sim}\left(u_{i}, v_{i}\right)$.

For each $i$ one of the vectors $\overrightarrow{p u}_{i}, \overrightarrow{p v}_{i}$ is the translation vector and thus

VOL. VI DÉDIÉ À M. CASIMIR KURATOWSKI it has the length $\sqrt{m}$ where $m$ is an integer. Since $L$ is bounded, there is only a finite number of lengths possible for $\overrightarrow{p u}_{i}$ or $\overrightarrow{p v}$. Therefore we may assume (restricting ourselves to a subsequence $\left\{\left(u_{i_{k}}, v_{i_{k}}\right)\right\}$ ) that for some $m$ all points $u_{i}$ or all points $v_{i}$ lie on a circle of radius $\sqrt{m}$. We can assume also that there exist

$$
\lim _{i \rightarrow \infty} u_{i}=u \quad \text { and } \quad \lim _{i \rightarrow \infty} v_{i}=v .
$$

Then, for sufficiently large $i,(u, v) \cap\left(u_{i}, v_{i}\right)=\left(u_{i}, v\right)$ or $\left(u, v_{i}\right)$. Since the second of these possibilities can be reduced to the first by changing the sense on $L$, we can assume that for every $i$

$$
(u, v) \cap\left(u_{i}, v_{i}\right)=\left(u_{i}, v\right) .
$$

Since the $\operatorname{arcs}\left(u_{i}, v_{i}\right)$ are congruent one to another by translations, each of these arcs is congruent by translation to $(u, v)$. Consequently (4) implies that $(u, v) \approx\left(u_{i}, v\right)$ for each $i$. Thus from $\left(\mathrm{P}_{2}\right)$ follows that $(u, v)$ is a segment. But then $\left(u_{i}, v_{i}\right)$ are segments of a straight line. This is impossible since we obtained that all points $u_{i}$ or all points $v_{i}$ lie on a circle. Thus the assumption that II does not hold leads to a contradiction.

MATHEMATICAL INSTITUTE OF THE POLISH ACADEMY OF SCIENCES

$$
\text { Rę̧u par la Rédaction le 17.1.1958 }
$$

SUR L'EXISTENCE DES INTEGRALES ASYMPTOTIQUES DES EQUATIONS DIFFERENTIELLES ISSUES D'UN ENSEMBLE DE DIMENSION ZÉRO

PAR

J. SZARSKI ET T. WAŻEWSKI (CRACOVIE)

§1. Nous dirons que l'ensemble $Z$ situé dans l'espace cartésien à trois dimensions $E^{3}$ est du type d'Antoine lorsque

$1^{\circ} Z$ est compact et contient au moins deux points différents,

$2^{\circ} Z$ ne contient ancun sous-ensemble connexe ne se réduisant pas à un point,

$3^{\circ}$ Si $K$ est un ensemble homéomorphe à la sphère $x^{2}+y^{2}+z^{2} \leqslant 1$ et si un point de $Z$ est situé à l'intérieur de $K$ tandis qu'un autre point de $Z$ est situé à l'extérieur de $K$, alors $Z$ possède au moins un point commun avec la frontière de $K$.

Remarque 1. L'exemple d'un tel ensemble $Z$ est dû à $L$. Antoine (cf. [1], p. 91-94). La dimension de cet ensemble est égale à zéro.

Remarque 2. On peut facilement démontrer que la projection orthogonale ou oblique d'un ensemble de ce type sur un plan quelconque constitue un continu. On peut pareillement démontrer que la projection ,,curviligne" de $Z$, effectuée le long des intégrales d'un système d'équations différentielles de la forme (1) sur une surface simplement connexe (suffisamment régulière et non tangente aux intégrales), constitue un continu.

§ 2. La note présente apporte une condition suffisante pour l'existence des intégrales d'un système de deux équations différentielles,

$$
d x / d t=f(x, y, t), \quad d y / d t=g(x, y, t),
$$

qui commencent sur un ensemble $Z$ du type d'Antoine et possèdent une certaine propriété asymptotique (sont asymptotiques par rapport à un tuyau $T)$. En appliquant la Remarque 2, nous montrons que l'on peut remplacer l'ensemble $Z$ par un ensemble connexe $Z_{1}$, ce qui permet d'employer la méthode topologique du travail [2].

En remplaçant dans le théorème le tuyau $T$ par un tuyau convenablement modifié, on peut obtenir des théorèmes relatifs à l'existence 
des intégrales commencant sur un ensemble du type d'Antoine et possédant différentes propriétés asymptotiques au sens classique.

Dans cet ordre d'idées, il est intéressant de rappeler qu'en appliquant le théorème du travail [3], on peut prouver que la famille d'intégrales asymptotiques relativement à $T$ dépend d'un paramètre au moins, conformément à un lemme de $K$. Borsuk et $K$. Kuratowski.

\$ 3. Relativement au système de deux équations différentielles (1), nous introduisons l'hypothèse suivante (cf. [2], p. 282):

HYPOTHEेSE H. I. Les fonctions $f$ et $g$ sont continues dans un ensemble ouvert $W$ contenu dans l'espace cartésien réel $E^{3}$ à trois dimensions, et par chaque point de $W$ il passe une intégrale unique du système (1.).

II. L'ensemble ouvert $T$

$$
|x|<1, \quad|y|<1, \quad-\infty<t<+\infty
$$

(dit dans la suite tuyau $T$ ), ainsi que sa frontière composée d'ensembles $A, B_{1}, B_{2}$ :

$$
\begin{array}{llll}
|x|=1, & |y| \leqslant 1, & -\infty<t<+\infty & \text { (ensemble } A), \\
|x| \leqslant 1, & y=1, & -\infty<t<+\infty & \text { (ensemble } \left.B_{1}\right), \\
|x| \leqslant 1, & y=-1, & -\infty<t<+\infty & \text { (ensemble } \left.B_{2}\right),
\end{array}
$$

sont contenus dans $W$; e'est-à-dire $T+A+B_{1}+B_{2} \subset W$.

III. Qn a les inégalités

$$
x f(x, y, t)<0 \quad \text { lorsque } \quad(x, y, t) \in A,
$$

(3) $\quad y g(x, y, t)>0 \quad$ lorsque $\left.\quad(x, y, t) \in B=B_{1}+B_{2}{ }^{1}\right)$.

IV. Pour l'ensemble $Z$, on a $Z \subset T+B_{1}+B_{2}-A, Z\left(B_{1}-A\right) \neq 0$, $Z\left(B_{2}-A\right) \neq 0$

V. L'ensemble $Z$ est du type d'Antoine (cf. §1).

THÉORÈmte. Dans l'hypothèse $\mathrm{H}$, l'ensemble $Z$ contient au moins un point $P$ asymptotique par rapport $\grave{a}(1)$ et $\grave{a} T$, e'est-à-dire tel que la demi-intégrale à droite de (1), issue de $P$ et prolongée d̀ droite dans $W$ aussi loin

(1) Les inégalités (2) et (3) expriment que par l'ensemble $A-B$ les intégrales de (1) entrent dans le tuyau $T$ et par $B-A$ elles en sortent (au sens strict), tandis qu'aux points de $A \cdot B$ elles glissent extérieurement à $T$ (cf. [2], p. 280). que possible, est asymptotique par rapport à $T$ (c'est-à-dire ne quitte jamais le tuyau $T$ ).

Démonstration. Choisissons $k$ de facon que l'ensemble $Z$ soit situé totalement à droite du plan $\Pi: t=k$, et désignons par $U$ la partie de $T$ située à droite de $I I$. Désignons par $\bar{U}$ la fermeture de $U$ et posons

$$
D=\left(T+B_{1}+B_{2}+A\right) \Pi=\bar{U} \Pi .
$$

En vertu de (2) et (3), la frontière du tuyau $U$ est composée de points de sortie stricte $\left({ }^{2}\right)$ (ensemble $\bar{C}\left(B_{1}+B_{2}-A-D\right)$ ), de points d'entrée stricte (ensemble $\bar{U}\left(A+D-B_{1}-B_{2}\right)$ ) et de points de glissement extérieur (ensemble $\bar{U}[A+D]\left[B_{1}+B_{2}\right]$ ).

Soit $M \in Z$ et désignons par $J(M)$ l'intégrale issue de $M$. Soit $G(M)$ le premier point en lequel, en s'avançant sur $J(M)$ à partir de $M$ vers la gauche, on rencontre l'ensemble $A+D-B_{1}-B_{2}$ et soit $Q(M)$ l'arc partiel fermé de $J(M)$ dont les extrémités sont les points $M I$ et $G(M)$. Si $M$ varie dans $Z$, le point $G(M)$ décrit un continu $K \subset A+D-B_{1}-B_{2}$ (cf. Remarque 2). En même temps que $M$ varie dans $Z$, l'arc $Q(M)$ engendre un ensemble $Z_{1}$ qui est aussi connexe, car l'extrémité $G^{\prime}(M)$ de $Q(M)$ varie dans le continu $K$. L'ensemble $Z_{1}$ est donc un continu, car il est connexe, borné et fermé. On a $Z+K \subset Z_{1}$. Chaque intégrale de (1), issue d'un point quelconque $R \in Z_{1}$ et prolongée vers la droite, rencontre évidemment l'ensemble $Z$. Le tuyau $U$, introduit auxiliairement dans ce qui précède, n'interviendra plus dans la suite. Afin de démontrer notre théorème, il suffit donc de prouver que $Z_{1}$ contient au moins un point asymptotique par rapport à (1) et à $T$. Posons

L'ensemble

$$
Z_{2}=Z_{1}+\left(B_{1}+B_{2}-A\right) .
$$

représente à la fois l'ensemble des points de sortie et celni de sortie stricte relativement à (1) et à $T$ (cf. [2], p. 280).

On a $Z_{2} S=S$. Cet ensemble est un rétracte de $S$, mais il n'est pas un rétracte de $Z_{2}$, car $Z_{2}$ est connexe, tandis que $S$ ne l'est pas. En vertu d'un théorème établi dans [2] (p. 303), l'ensemble $Z_{2}$ contient au moins un point $P$ asymptotique par rapport à (1) et à $T$. Mais $P$ ne peut évidemment pas appartenir à $S$. En vertu de (4) et (5), il en résulte que $P \in Z_{1}$, ce qui termine la démonstration.

$\left({ }^{2}\right)$ Pour ees notions ef. [2], p. 280 
[1] L. Antoine, Sur l'homéomorphie de deux figures et de leurs voisinages, Thèses présentées à la Faculté des Sciences de Strasbourg, Paris 1921, p. 1-101.

[2] T. Ważewski, Sur un principe topologique de l'examen de l'allure asymptotique des intégrales des équations différentielles ordinaires, Annales de la Société Polonaise de Matlématique 20 (1947), p. 279-313.

[3] - Sur l'évaluation du nombre des paramètres essenticls dont dépend la famille des intégrales d'un système d'équations différentitlles ayant une propriété asymptotique, Bulletin de l'Académie Polonaise des Sciences, Classe III, 1 (1953), p. 3-5.

Reçu par la Rédaction le 20.1.1958

DÉDIÉ À M. CASIMIR KURATOWSKI

1958

\section{REMARQUE SUR LA STRUCTURE DE L'ENSEMBLE ENGENDRE PAR LES INTÉGRALES ASYMPTOTIQUES D'UN SYSTEMME D'ÉQUATIONS DIFFÉRENTIELLES}

PAR

Z. MIKOEAJSKA (CRACOVIE)

Considérons le système de $p+q$ équations différentielles écrit sous la forme vectorielle

$$
\frac{d X}{d t}=F(X, Y), \quad \frac{d Y}{d t}=G(X, Y),
$$

où $X=\left(x_{1}, \ldots, x_{p}\right), Y=\left(y_{1}, \ldots, y_{q}\right), F=\left(f_{1}, \ldots, f_{p}\right), G=\left(g_{1}, \ldots, g_{q}\right)$, $f_{i}=f_{i}(X, Y), g_{i}=g_{i}(X, Y)$.

Posons $|X|=\left(x_{1}^{2}+\ldots+x_{p}^{2}\right)^{1 / 2},|Y|=\left(y_{1}^{2}+\ldots+y_{p}^{2}\right)^{1 / 2}$. Relativement au système (1), nous introduisons l'hypothèse suivante:

Hүротнѐse H. $F$ et $G$ sont de classe $O^{1}$ dans un ensemble ouvert $W$.

Pour l'ensemble $R$

$$
|X| \leqslant a, \quad|Y| \leqslant b \quad(0<a<\infty, 0<b<\infty)
$$

on a par hypothèse $R \subset W$.

La frontière $F$ de $R$ se compose, par hypothèse, d'ensemble $S$ des points de sortie stricte

$$
|X|<a, \quad|Y|=b,
$$

d'ensemble $E$ des points d'entrée stricte

$$
|X|=a, \quad|Y|<b,
$$

et d'ensemble $M$ des points de glissement extérieur

$$
|X|=a, \quad|Y|=b .
$$

Remarque 1. Pour la définition des points de sortie stricte, d'entrée stricte et de glissement extérieur, cf. [1] (p. 280).

Les ensembles $S, E$ et $M$ se composent respectivement de points de sortie stricte, d'entrée stricte et de glissement extérieur, si l'on suppose 